\title{
FTY720 and cisplatin synergistically induce the death of cisplatin-resistant melanoma cells through the downregulation of the PI3K pathway and the decrease in epidermal growth factor receptor expression
}

\author{
ASAKO ISHITSUKA, ETSUKO FUJINE, YOKO MIZUTANI, CHISATO TAWADA, \\ HIROYUKI KANOH, YOSHIKO BANNO and MARIKO SEISHIMA \\ Department of Dermatology, Gifu University Graduate School of Medicine, 1-1 Yanagido, Gifu 501-1194, Japan
}

Received May 16, 2014; Accepted July 31, 2014

DOI: $10.3892 /$ ijmm.2014.1882

\begin{abstract}
Sphingosine kinase (SK), a key enzyme in sphingosine-1-phosphate (S1P) synthesis, is known to be overexpressed in various types of cancer cells. The effects of anticancer agents on SK1/S1P signaling have not yet been fully assessed in melanoma cells. In the present study, we investigated the effects of the combination of FTY720, an S1P receptor antagonist, and cisplatin, a DNA-damaging agent, on the induction of the death of human melanoma cells, as well as the molecular mechanisms involved. The viability of various human melanoma cell lines was examined following treatment with anticancer drugs. The cisplatin-resistant SK-Mel-28 and cisplatin-sensitive A375 cell lines were selected for this analysis. Protein expression and apoptotic rates were evaluated by western blot analysis following treatment with cisplatin and/or FTY720. Following treatment with a combination of FTY720 and cisplatin, cell viability significantly decreased and the expression of apoptosis-associated cleaved poly(ADP-ribose) polymerase (PARP) was significantly higher in comparison to treatment with cisplatin alone in the SK-Mel-28 cells. In addition, the combination of FTY720 and cisplatin reduced the protein expression of SK1 and the phosphorylation levels of phosphoinositide 3-kinase (PI3K), Akt and mTOR in the SK-Mel-28 cells; the expression of epidermal growth factor receptor (EGFR) was also markedly reduced. These findings suggest that FTY720 and cisplatin synergistically induce cell death through the downregulation of the PI3K/Akt/mTOR pathway and the decrease in EGFR expression in SK-Mel-28 cells. Thus, the combination of FTY720 and cisplatin may have therapeutic potential for chemotherapy-resistant melanoma,
\end{abstract}

Correspondence to: Asako Ishitsuka, Department of Dermatology, Gifu University Graduate School of Medicine, 1-1 Yanagido, Gifu 501-1194, Japan

E-mail: ishitsuk@gifu-u.ac.jp

Key words: cisplatin, epidermal growth factor receptor, FTY720, melanoma, sphingosine kinase 1, sphingosine 1-phosphate and the effects are likely exerted through the downregulation of S1P signaling.

\section{Introduction}

Sphingolipids are the main components of lipid rafts and have critical functions as signaling molecules. Sphingosine 1-phosphate $(\mathrm{S} 1 \mathrm{P})$ and ceramide regulate proliferation and apoptosis (1-4). In response to various stimuli, ceramide mediates cell death and apoptosis, whereas S1P abrogates apoptosis and mediates cell proliferation and migration (5). Sphingosine kinase 1 (SK1) is the key enzyme responsible for converting sphingosine to S1P. The signaling pathway via the S1P receptor contributes to cancer cell survival and proliferation (6), apoptosis reduction (7) and oncogenic transformation (8). Various types of cancer cells show high levels of SK1 expression/ activity, increasing their resistance to anticancer agents, such as anthracyclines, doxorubicin and camptothecin $(9,10)$. Thus, SK1 may play an important role in the development and proliferation of cancers, such as melanoma (11-13). FTY720 (fingolimod) is phosphorylated by SK1 and SK2 (14), and acts as an S1P receptor antagonist $(14,15)$. FTY720 induces apoptosis in prostate (15), liver ( 7$)$, bladder cancer $(12)$ and melanoma $(16,17)$ by the direct inhibition of SK1 (18). In the present study, we investigated the combined effects of FTY720 and cisplatin, a DNA damaging agent, on cell death, SK1 expression, S1P signaling and epidermal growth factor receptor (EGFR) expression in anticancer drug-resistant melanoma cells.

\section{Materials and methods}

Chemical reagents. The S1P receptor antagonist, FTY720, was obtained from Cayman Chemical Co. (Ann Arbor, MI, USA). Cisplatin, carboplatin, paclitaxel, dacarbazine, bovine serum albumin, Dulbecco's modified Eagle's medium (DMEM) and Eagle's minimal essential medium (EMEM) were purchased from Wako Pure Chemical Industries (Osaka, Japan). RPMI1640, non-essential amino acids (NEAA) and dimethyl sulfoxide (DMSO) were obtained from Sigma-Aldrich (St. Louis, MO, USA). Rabbit polyclonal antibodies against 

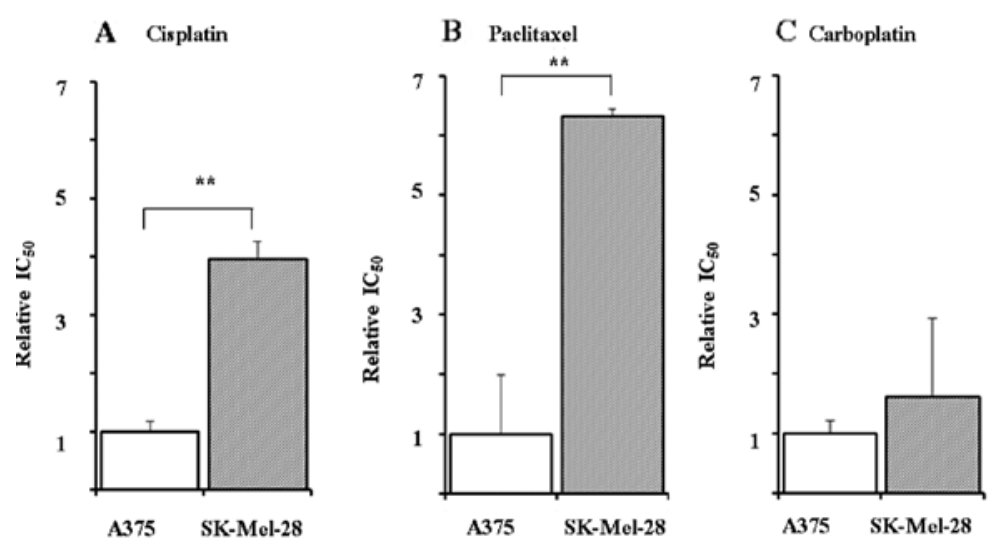

Figure 1. In the SK-Mel-28 cells and A375 cells, the 50\% inhibitory concentrations $\left(\mathrm{IC}_{50}\right)$ for various anticancer drugs were examined by MTT assay. These cell lines $\left(5 \times 10^{3}\right.$ cells $/$ well) were cultured in 96 -well plates for $24 \mathrm{~h}$, and were then treated with various concentrations of (A) cisplatin, (B) paclitaxel, or (C) carboplatin for $48 \mathrm{~h}$. Each condition was measured in triplicate, and the ratio of the $\mathrm{IC}_{50}$ value in the SK-Mel-28 cells to that of the A375 was calculated. Data are expressed as the means $\pm \mathrm{SD}$ of 3 independent experiments. ${ }^{* *} \mathrm{P}<0.01$.

SK1, p53, cleaved poly(ADP-ribose) polymerase (PARP), phosphoinositide 3-kinase (PI3K), phosphorylated (p)-PI3K, Akt, p-Akt, mammalian target of rapamycin (mTOR), p-mTOR, EGFR and p-EGFR were obtained from Cell Signaling Technology (Beverly, MA, USA) and rabbit polyclonal antibody against SK2 was obtained from Abcam (Cambridge, UK).

Cell lines and measurement of cell viability. The human melanoma SK-Mel-28 cells were obtained from the JCRB Cell Bank (Osaka, Japan), the human melanoma cell lines (A375, A2058 and WM115) and the mouse melanoma B16 cells were purchased from the European Collection of Cell Cultures (ECACC). The SK-Mel-28 and WM115 cells were cultured in RPMI-1640 containing 10\% fetal bovine serum (FBS) and $0.1 \%$ tyrosine. The A375 and B16 cells were cultured in DMEM containing 10\% FBS. The A2058 cells were grown in EMEM containing $1 \%$ of NEAA and $10 \%$ FBS. To assess viability, melanoma cells were plated at $1 \times 10^{4}$ cells/well and $5 \times 10^{3}$ cells/ well in 24- and 96-well plates, respectively. The anticancer drugs were dissolved in DMSO. When the determination of $\mathrm{IC}_{50}$ was performed in 96-well plates, cisplatin, paclitaxel, carboplatin and dacarbazine were added at doses of up to 50, 10,350 and $750 \mu \mathrm{M}$, respectively. In 24-well plates, the cells were treated with up to $150 \mu \mathrm{M}$ of cisplatin. The cells treated with DMSO only were used as controls. Viability was analyzed following $48 \mathrm{~h}$ of treatment with 3-(4,5-dimethylthiazol-2-yl)2,5-diphenyl-tetrazolium bromide (MTT) using a standard assay, and $50 \%$ inhibitory concentrations $\left(\mathrm{IC}_{50}\right)$ were calculated.

Western blot analysis. The cells were lysed by sonication in lysis buffer (1\% Nonidet P-40, $0.5 \%$ sodium cholate, $1 \%$ SDS, $1 \mathrm{mM}$ EDTA, $1 \mathrm{mM}$ EGTA, $150 \mathrm{mM} \mathrm{NaCl}, 20 \mathrm{mM}$ HEPES, $3 \mathrm{mM}$ $\mathrm{MgCl}_{2}$, protease inhibitors, $0.5 \mathrm{mM} 4$-deoxypyridoxine, $20 \mathrm{mM}$ $\beta$-glycerophosphate, $1 \mathrm{mM}$ sodium fluoride and $1 \mathrm{mM}$ sodium orthovanadate, $\mathrm{pH} 7.4)$. Total cell lysates ( $20 \mu \mathrm{g}$ protein) were separated by electrophoresis on 10-7.5\% SDS-polyacrylamide gels and transferred onto polyvinylidene difluoride membranes (Millipore, Danvers, MA, USA). The membranes were blocked with $5 \%$ bovine serum albumin. The expression of SK1, SK2, cleaved PARP, total PI3K, p-PI3K, total Akt, p-Akt, total mTOR, p-mTOR, total EGFR and p-EGFR was measured by western blot analysis. Anti- $\beta$-actin antibody was used as the loading control. Followign repeated washes, bound antibodies were detected using the ECL Western Blotting Detection system (GE Healthcare Ltd., Buckinghamshire, UK). Protein band density was determined using a densitometer (Multi Gauge, version 3.1; Fuji Film, Tokyo, Japan).

Statistical analysis. Data are expressed as the means \pm SD and compared using the Student's t-test. P-values $<0.05$ were considered to indicate statistically significant differences.

\section{Results}

Changes in cell viability induced by anticancer drugs. We examined the sensitivity of 5 melanoma cell lines (i.e., SK-Mel-28, A375, WM115, A2058 and B16) to 4 anticancer drugs (cisplatin, paclitaxel, carboplatin and dacarbazine). The $\mathrm{IC}_{50}$ value for cisplatin was the highest in the SK-Mel-28 cells and the lowest in the A375 cells (data not shown). The relative $\mathrm{IC}_{50}$ values for cisplatin, paclitaxel and carboplatin in the SK-Mel-28 cells vs. the A375 cells were 3.97-, 6.33- and 1.62-fold greater, respectively (Fig. 1). The $\mathrm{IC}_{50}$ value for dacarbazine was $>20$-fold greater than that of cisplatin in the SK-Mel-28 cells (data not shown). Therefore, for further analysis, the SK-Mel-28 and A375 cells were used as cisplatin-resistant and cisplatin-sensitive cell lines, respectively.

Effects of FTY720 on viability of cisplatin-resistant cells. We investigated the effects of FTY720 on the viability of the anticancer drug-resistant cell line, SK-Mel-28. In the SK-Mel-28 cells treated with cisplatin, the $\mathrm{IC}_{50}$ value was reduced from 85.9 to $54.2 \mu \mathrm{M}$ in the presence of $3 \mu \mathrm{M}$ FTY720 (an approximate $37 \%$ reduction) (Fig. 2). The $\mathrm{IC}_{50}$ value for cisplatin in the A375 cells $(1.3 \mu \mathrm{M})$ was lower than that in the SK-Mel-28 cells and the combination with FTY720 had no effects on the $\mathrm{IC}_{50}$ value for cisplatin in the $\mathrm{A} 375$ cells (data not shown). Treatment with FTY720 alone at $3 \mu \mathrm{M}$ had no significant effect on cell viability, although treatment with $7 \mu$ M FTY720 reduced viability to $10 \%$ of the control (data not shown). These results suggest that the combinatio of of FTY720 and cisplatin significantly reduces viability in comparison to treatment with cisplatin alone in SK-Mel-28 cells. 


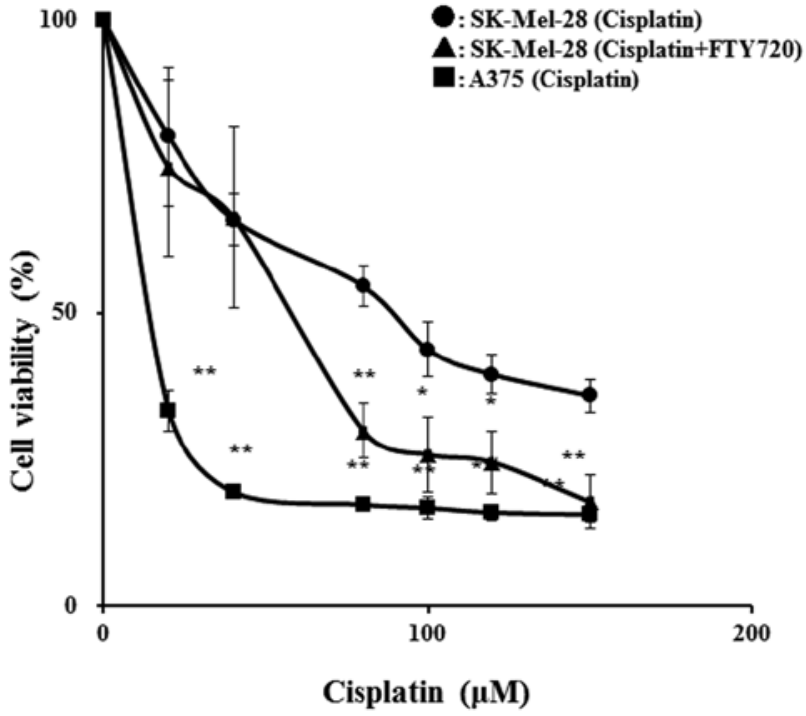

Figure 2. Effects of FTY720 on the viability of SK-Mel-28 cells. After the SK-Mel-28 or A375 cells ( $1 \times 10^{4}$ cells/well) were incubated with various concentrations of cisplatin in the presence or absence of $3 \mu \mathrm{M}$ FTY720 for $48 \mathrm{~h}$, MTT assays were performed. Each condition was measured in triplicate, and data are expressed as the means \pm SD of 3 independent experiments. Viability was reduced by the addition of FTY720 in the SK-Mel-28 cells. $\mathrm{P}<0.05,{ }^{* * *} \mathrm{P}<0.01$

Effects of FTY720 on apoptosis. We investigated the effects of FTY720 on the apoptosis of SK-Mel-28 and A375 cells. Cleaved PARP, a marker of apoptosis, was examined by western blot analysis. In the A375 cells, the expression of cleaved PARP significantly increased following treatment with cisplatin, but not following treatment with FTY720 alone (Fig. 3). Although PARP degradation increased in the SK-Mel-28 cells treated with cisplatin alone, combined treatment with FTY720 induced a synergistic increase in PARP degradation (Fig. 3). These results demonstrated that the combination of FTY720 and cisplatin enhanced apoptosis in comparison to treatment with cisplatin alone in the SK-Mel-28 cells, but not in the A375 cells.

Changes in SK1 and SK2 expression induced by FTY720. We investigated the changes in SK expression levels in the SK-Mel-28 cells following treatment with FTY720, cisplatin or a combination of FTY720 and cisplatin. Treatment with FTY720 or cisplatin alone produced no change in SK1 protein expression (Fig. 4A). Treatment with a combination of FTY720 and cisplatin for 24 and $48 \mathrm{~h}$ caused a synergistic decrease in SK1 expression, while SK2 expression remained unaltered (Fig. 4B). Thus, the combination of FTY720 and cisplatin mainly reduced SK1 expression in the SK-Mel-28 cells within $48 \mathrm{~h}$.

Downregulation of SIP signaling and the decrease in EGFR expression following treatment with a combination of FTY720 and cisplatin. To investigate the effects of FTY720 on signal transduction via the S1P receptor, the expression levels of total and phosphorylated forms of PI3K, Akt and mTOR were examined by western blot analysis in the SK-Mel-28 cells following treatment with FTY720, cisplatin alone, or a combination of FTY720 and cisplatin. The combination of FTY720 and cisplatin reduced the expression of the phosphorylated forms of PI3K,
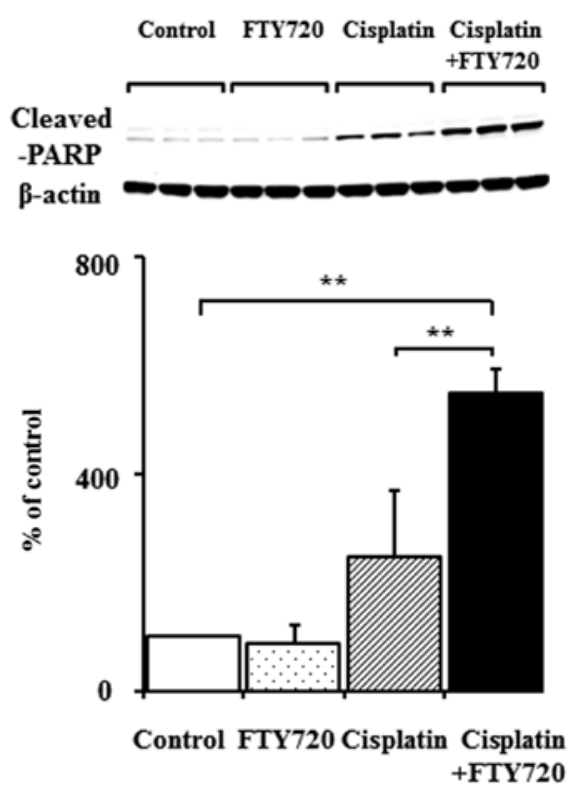

Figure 3. Effects of FTY720 on the apoptosis of SK-Mel-28 and A375 cells. SK-Mel-28 and A375 cells were treated with $3 \mu \mathrm{M}$ FTY720 and $30 \mu \mathrm{M}$ cisplatin, alone or in combination for $48 \mathrm{~h}$. Cell lysates were subjected to western blot analysis with antibody against cleaved PARP, and the band intensity was measured. Results are expressed as a percentage increase relative to the untreated controls. Data are expressed as the means $\pm \mathrm{SD}$. ${ }^{* *} \mathrm{P}<0.01$.

Akt and mTOR, but not the total protein expression (Fig. 5A-C). Unexpectedly, the expression of total and p-ERK remained unaltered following combination treatment (data not shown). Cross-talk between SK1/S1P and EGFR-dependent pathways has been previously reported (19-21). Subsequently, we examined the effects of FTY720 on EGFR expression in the SK-Mel-28 cells. As shown in Fig. 5D, total EGFR expression was decreased following treatment with a combination of FTY720 and cisplatin, whereas total EGFR expression remained unaltered following treatment with cisplatin and FTY720 alone.

\section{Discussion}

Combination chemotherapy is an important strategy for improving therapeutic outcomes and reducing the side-effects of anticancer drugs. Various studies have indicated the possible utility of combining anticancer drugs with agents associated with the SK1/S1P pathway for cancer therapy (22-24). The combination of SK inhibitors, including siRNA, with anticancer drugs has been shown to be effective against drug-resistant colon cancer $(22,23)$ and pancreatic cancer $(24)$. The combination of doxorubicin and SK1-locked acid-antisense oligonucleotides has also been shown to be effective against gastric cancer (25). Recent studies have indicated that FTY720, which is phosphorylated by SK and acts as an antagonist of S1P, may enhance the efficacy of anticancer therapies. This compound has been shown to induce dose-dependent cell death in several human tumor cell lines, such as prostate and bladder cancer, as well as hepatocellular carcinoma $(7,12,15)$. Moreover, FTY720 has been shown to reduce tumor growth and metastasis by increasing radiotherapeutic sensitivity in prostate cancer (26). In murine melanoma, FTY720 reduces the development of metastatic melanoma in vivo and apoptosis in tumor cells (16). Furthermore, FTY720 

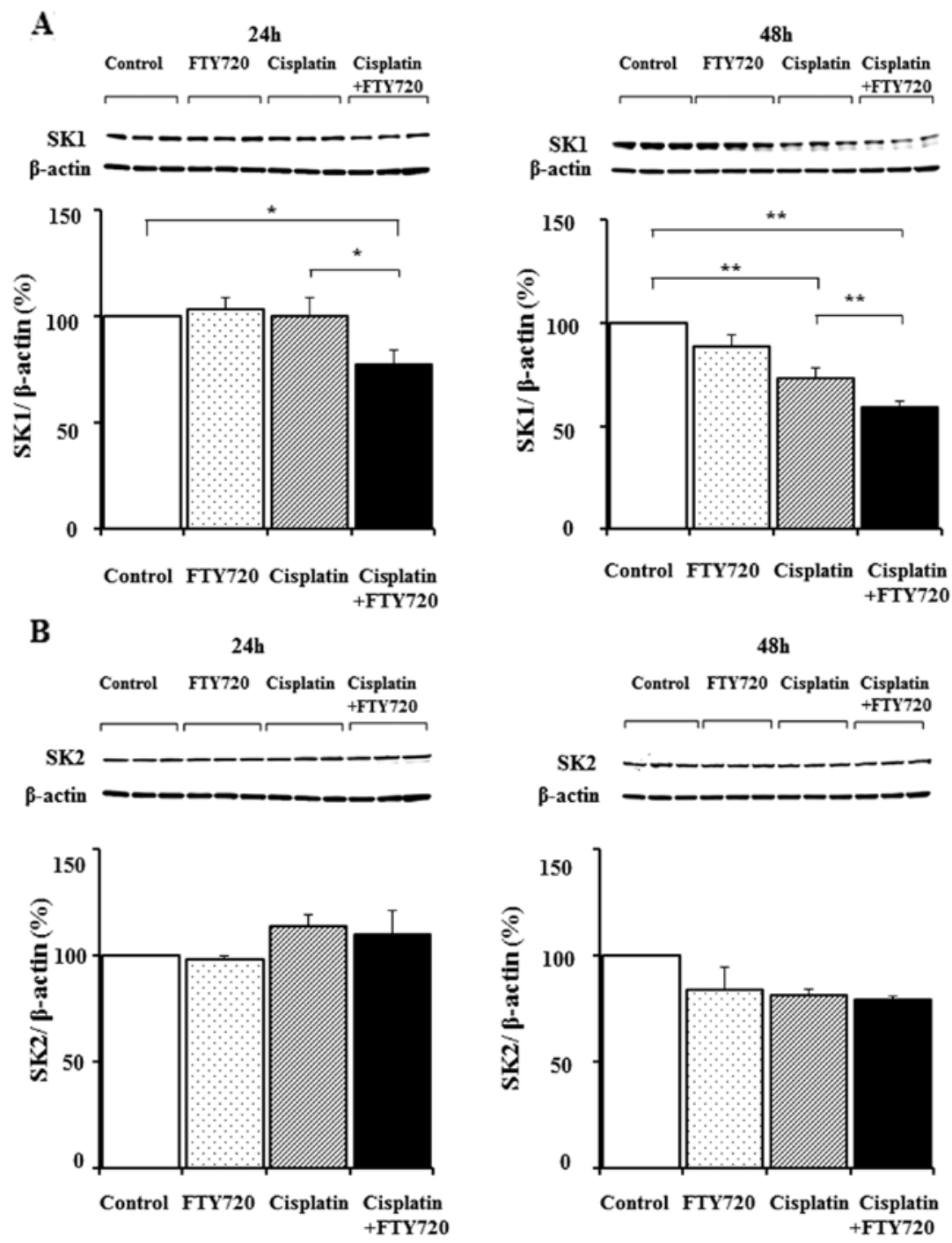

Figure 4. Effects of FTY720 and cisplatin on the expression of (A) sphingosine kinase (SK)1 and (B) SK2 in SK-Mel-28 cells. SK-Mel-28 cells were treated with $3 \mu \mathrm{M}$ FTY 720 and $30 \mu \mathrm{M}$ cisplatin, alone or in combination for 24 and $48 \mathrm{~h}$. Cell lysates were subjected to western blot analysis with antibodies against $\mathrm{SK} 1$ and SK2, with $\beta$-actin as the loading control. Each condition was examined in triplicate, and data are expressed as the means $\pm \mathrm{SD}$. ${ }^{*} \mathrm{P}<0.05$.

has been shown to inhibit S1P- and VEGF-induced angiogenesis and tumor vascularization in a murine model of melanoma cells (17). These studies suggest that functional antagonism between S1P receptors and FTY720 inhibits angiogenesis and provides a novel anticancer drug in chemotherapy. However, the precise mechanisms underlying the induction of cell death have not yet been elucidated. In this study, we demonstrated that the SK-Mel-28 cell line was the most resistant to anticancer drugs, such as cisplatin (Fig. 1), and that the combination of FTY720 and cisplatin significantly reduced viability in this cell line (Fig. 2). The viability of the SK-Mel-28 cells remained unaltered by low concentrations of FTY720 alone, but treatment with over $7 \mu \mathrm{M}$ FTY720 induced cell death (data not shown). Several mechanisms have been proposed to explain the anticancer efficacy of FTY720; for instance, it induces apoptosis in various types of cancer $(9,12,16,17,25)$ through the direct inhibition and proteasomal degradation of SK 1 at a high concentration $(10 \mu \mathrm{M})$ in human prostate cancer cells (18). Other studies have indicated that SK1 is downregulated by genotoxic stress and proteolysis of p53 induced by caspases $(27,28)$. The resistant melanoma cell line, SK-Mel-28, harbors a p53 mutation, and cell death induced by anticancer drugs is independent of p53 (29). In the present study, the expression of SK1, but not that of SK2, in the SK-Mel-28 cells was reduced following treatment with a combination of FTY720 and cisplatin, but not following treatment with cisplatin or FTY720 alone (Fig. 4). Combination treatment with FTY720 and cisplatin likely induced SK1 degradation by p53-independent caspase activation, resulting in a significant increase in PARP cleavage and the induction of apoptosis. Further studies required to investigate the involvement of SK2 in melanoma, as the roles of SK2 in cancer cells have not yet been elucidated.

Moreover, we investigated the molecular mechanisms underlying the induction of apoptosis following treatment with a combination of FTY720 and cisplatin in the SK-Mel-28 cells. Previous studies have demonstrated that the interaction between FTY720 and the $\mathrm{S}_{1} \mathrm{P}_{1}$ receptor blocks STAT3 signaling in B-cell lymphoma (30) and the FTY-720-induced apoptosis of renal cancer cells is mediated by the inhibition of ERK activation (13). Apoptosis induced by chemotherapeutic agents in SK-Mel-28 


\section{A $\mathrm{PI} 3 \mathrm{~K}$}

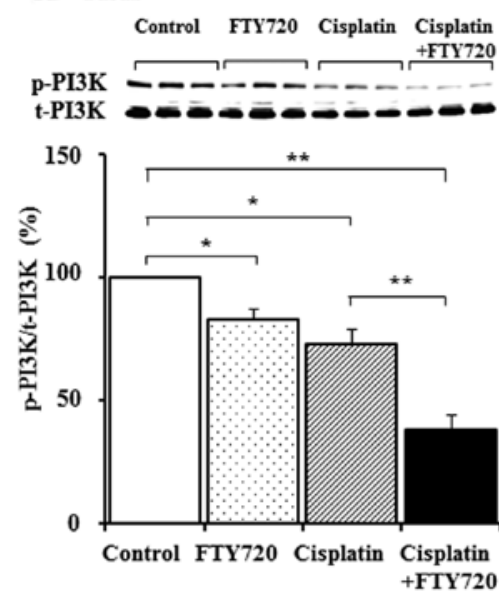

C ${ }_{\mathrm{mTOR}}$
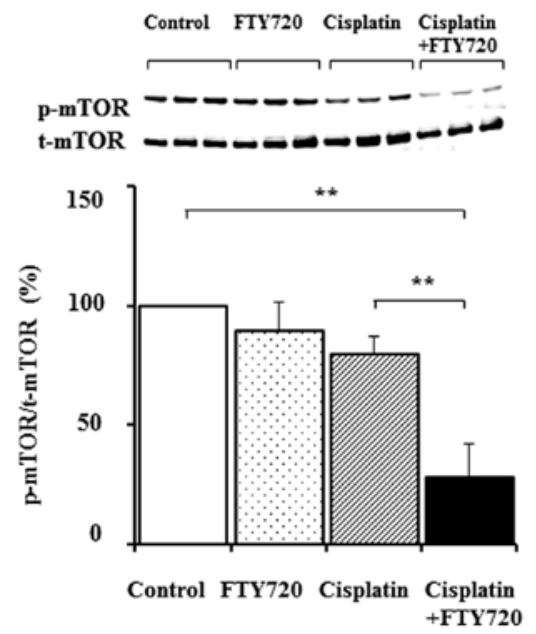

B Akt
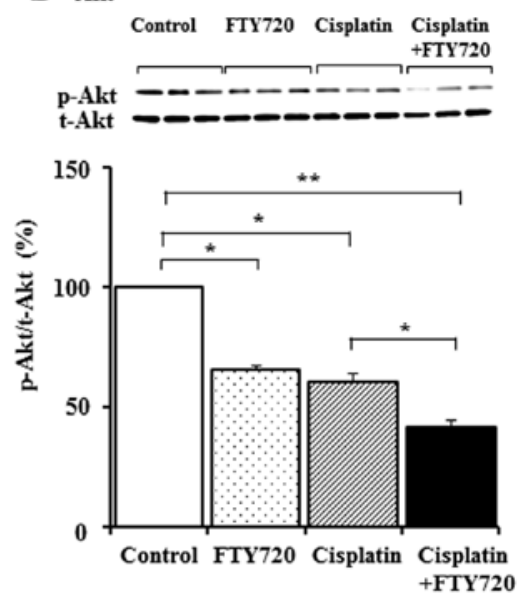

D EGFR
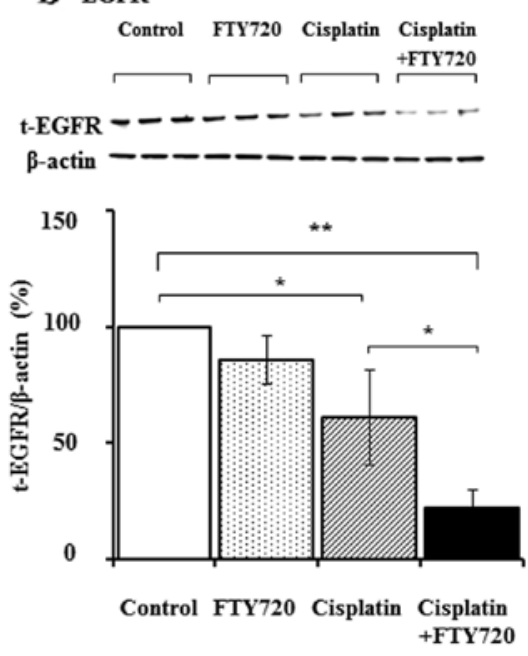

Figure 5. Effects of FTY720 and cisplatin on signaling transduction in SK-Mel-28 cells. SK-Mel-28 cells were treated with $3 \mu \mathrm{M}$ of FTY720 and $30 \mu \mathrm{M}$ cisplatin, alone or in combination; for $48 \mathrm{~h}$, and cell lysates were subjected to western blot analysis with antibodies to total (t) and phosphorylated (p) forms of (A) PI3K, (B) Akt, (C) mTOR and (D) EGFR. The ratios of phosphorylated to total PI3K, Akt, mTOR were calculated. The expression of EGFR is shown as the ratio of total-EGFR to $\beta$-actin. Results are expressed as ratios of treated to untreated cells. Each condition was examined in triplicate, and data are expressed as the means \pm SD. ${ }^{*} \mathrm{P}<0.05,{ }^{* *} \mathrm{P}<0.01$.

cells is mediated by the activation of stress kinases, such as p38 MAP kinase and SAPK/JNK (29), and by the increased expression of the transactivation factor, E2F-1 (31). Furthermore, accumulating evidence suggests that the PI3K/Akt pathway is related to chemoresistance, mainly through escape from apoptosis, and these signaling pathways promote cell survival and proliferation (32). The MAPK pathway via ras-raf-MEKERK and the PI3K-Akt pathway are major pathways in cancer development and progression (33), and the latter is particularly important in cell survival in melanoma (34). Our results revealed that combination treatment with FTY720 and cisplatin reduced the levels of pp-PI3K and p-Akt (Fig. 5). However, this treatment did not reduce ERK activation, suggesting that signal transduction via the PI3K/Akt pathway through the $\mathrm{S}_{1} \mathrm{P}_{1}$ receptor was suppressed by FTY720. Furthermore, mTOR, which belongs to the PI3K family, regulates cell growth and survival via p70S6 kinase activation in cancer cells $(35,36)$. Therefore, studies have noted that the inhibition of mTOR reduces the proliferation of colorectal cancer, prostate cancer and esophageal squamous cell carcinoma (37). In the present study, we demonstrated that the combination of FTY720 and cisplatin, but not cisplatin alone, suppressed p-mTOR levels (Fig. 5). Thus, FTY720 may inhibit the PI3K/Akt/mTOR pathway in cancer therapy. We also demonstrated that the combination of FTY720 and cisplatin reduced EGFR expression in the SK-Mel-28 cells (Fig. 5). It is well known that estrogen induces S1P release via SK1 activation, and that the subsequent activation of the $\mathrm{S} 1 \mathrm{P}$ receptor $\mathrm{S}_{1} \mathrm{P}_{3}$, leads to receptor transactivation in human breast cancer (19). Such cross-talk between S1P/SK1 and EGFR has also been reported in gastric cancer (20) and human glioblastoma (21). Therefore, our results suggest that the transactivation of EGFR by S1P was also reduced by combination treatment with FTY720 in melanoma cells. EGFR expression is enhanced in various types of cancer cells and anti-EGFR drugs have been developed as anticancer agents (38). Thus, the decrease in EGFR expression may be an important mechanism through which the combination of FTY720 and cisplatin induces apoptosis in SK-Mel-28 cells.

In conclusion, compared to treatment with alone, combination treatment with FTY720 and cisplatin exerted synergistic effects on the induction of apoptosis in drug-resistant melanoma cells 
by SK1 degradation, possibly due to the downregulation of the $\mathrm{PI} 3 \mathrm{~K} / \mathrm{Akt} / \mathrm{mTOR}$ pathway via the $\mathrm{S} 1 \mathrm{P}$ receptor and the reduced EGFR expression. These results indicate that the inhibition of survival signals via the SK/S1P pathway may be a molecular target for cancer therapy, and FTY720 may serve as a useful agent in chemotherapy.

\section{Acknowledgements}

The present study was supported in part by Grants-in-Aid for Scientific Research (C) 24590185 from the Ministry of Education, Culture, Sports, Science and Technology of Japan.

\section{References}

1. Taha TA, Hannun, YA and Obeid LM: Sphingosine kinase: biochemical and cellular regulation and role in disease. J Biochem Mol Biol 39: 113-131, 2006.

2. Ogretmen B and Hannun YA: Biologically active sphingolipids in cancer pathogenesis and treatment. Nat Rev Cancer 4: 604-616, 2004.

3. Olivera A, Kohama T, Edsall LC, et al: Sphingosine kinase expression increases intracellular sphingosine-1-phosphate and promotes cell growth and survival. J Cell Biol 147: 545-558, 1999.

4. Xia P, Wang L, Gamble JR and Vadas MA: Activation of sphingosine kinase by tumor necrosis factor-alpha inhibits apoptosis in human endothelial cells. J Biol Chem 274: 34499-34505, 1999.

5. Spiegel S and Milstien S: Sphingosine-1-phosphate: an enigmatic signalling lipid. Nat Rev Mol Cell Biol 4: 397-407, 2003.

6. Xia P, Gamble JR, Wang L, et al: An oncogenic role of sphingosine kinase. Curr Biol 10: 1527-1530, 2000.

7 Ho JW, Man K, Sun CK, Lee TK, Poon RT and Fan ST: Effects of a novel immunomodulating agent, FTY720, on tumor growth and angiogenesis in hepatocellular carcinoma. Mol Cancer Ther 4: 1430-1438, 2005.

8 Pchejetski D, Doumerc N, Golzio M, et al: Chemosensitizing effects of sphingosine kinase-1 inhibition in prostate cancer cell and animal models. Mol Cancer Ther 7: 1836-1845, 2008.

9 French KJ, Schrecengost RS, Lee BD, et al: Discovery and evaluation of inhibitors of human sphingosine kinase. Cancer Res 63 5962-5969, 2003.

10. Shida D, Takabe K, Kapitonov D, Milstien S and Spiegel S: Targeting SphK1 as a new strategy against cancer. Curr Drug Targets 9: 662-673, 2008.

11. Vadas M, Xia P, McCaughan G and Gamble J: The role of sphingosine kinase 1 in cancer: oncogene or non-oncogene addiction? Biochim Biophys Acta 1781: 442-447, 2008.

12. Azuma H, Takahara S, Horie S, Muto S, Otsuki Y and Katsuoka Y: Induction of apoptosis in human bladder cancer cells in vitro and in vivo caused by FTY720 treatment. J Urol 169: 2372-2377, 2003

13. Ubai T, Azuma H, Kotake Y, et al: FTY720 induced Bcl-associated and Fas-independent apoptosis in human renal cancer cells in vitro and significantly reduced in vivo tumor growth in mouse xenograft. Anticancer Res 27: 75-88, 2007.

14. Billich A, Bornancin F, Dévay P, Mechtcheriakova D, Urtz N and Baumruker T: Phosphorylation of the immunomodulatory drug FTY720 by sphingosine kinases. J Biol Chem 278: 47408-47415, 2003.

15. Wang JD, Takahara S, Nonomura N, Ichimaru N, Toki K and Azuma H: Early induction of apoptosis in androgen-independent prostate cancer cell line by FTY720 requires caspase-3 activation. Prostate 40: 50-55, 1999.

16. Pereira FV, Arruda DC, Figueiredo CR, et al: FTY720 induces apoptosis in B16F10-NEX2 murine melanoma cells, limits metastatic development in vivo, and modulates the immune system. Clinics (Sao Paulo) 68: 1018-1027, 2013.

17. LaMontagne $\mathrm{K}$, Littlewood-Evans A, Schnell C, et al: Antagonism of sphingosine-1-phosphate receptors by FTY720 inhibits angiogenesis and tumor vascularization. Cancer Res 66 221-231, 2006.
18. Tonelli F, Lim KG, Loveridge C, et al: FTY720 and (S)-FTY720 vinylphosphonate inhibit sphingosine kinase 1 and promote its proteasomal degradation in human pulmonary artery smooth muscle, breast cancer and androgen-independent prostate cancer cells. Cell Signal 22: 1536-1542, 2010.

19. Sukocheva O, Wadham C, Holmes A, et al: Estrogen transactivates EGFR via the sphingosine 1-phosphate receptor Edg-3: the role of sphingosine kinase-1. J Cell Biol 173: 301-310, 2006.

20. Shida D, Fang X, Kordula T, et al: Cross-talk between LPA1 and epidermal growth factor receptors mediates up-regulation of sphingosine kinase 1 to promote gastric cancer cell motility and invasion. Cancer Res 68: 6569-6577, 2008.

21. Paugh BS, Paugh SW, Bryan L, et al: EGF regulates plasminogen activator inhibitor-1 (PAI-1) by a pathway involving c-Src, PKCdelta, and sphingosine kinase 1 in glioblastoma cells. FASEB J 22: 455-465, 2008.

22. Nemoto S, Nakamura M, Osawa Y, et al: Sphingosine kinase isoforms regulate oxaliplatin sensitivity of human colon cancer cells through ceramide accumulation and Akt activation. J Biol Chem 84: 10422-10432, 2009.

23. Kawahara S, Otsuji Y, Nakamura M, et al: Sphingosine kinase 1 plays a role in the upregulation of CD44 expression through extracellular signal-regulated kinase signaling in human colon cancer cells. Anticancer Drugs 24: 473-483, 2013.

24. Guillermet-Guibert J, Davenne L, Pchejetski D, et al: Targeting the sphingolipid metabolism to defeat pancreatic cancer cell resistance to the chemotherapeutic gemcitabine drug. Mol Cancer Ther 8: 809-820, 2009.

25. Fuereder T, Hoeflmayer D, Jaeger-Lansky A, et al: Sphingosine kinase 1 is a relevant molecular target in gastric cancer. Anticancer Drugs 22: 245-252, 2011.

26. Pchejetski D, Bohler T, Brizuela L, et al: FTY720 (fingolimod) sensitizes prostate cancer cells to radiotherapy by inhibition of sphingosine kinase-1. Cancer Res 70: 8651-8661, 2010.

27. Heffernan-Stroud LA, Helke KL, Jenkins RW, De Costa AM, Hannun YA and Obeid LM: Defining a role for sphingosine kinase 1 in p53-dependent tumors. Oncogene 31: 1166-1175, 2012.

28. Taha TA, Osta W, Kozhaya L, et al: Down-regulation of sphingosine kinase-1 by DNA damage: dependence on proteases and p53. J Biol Chem 279: 20546-20554, 2004.

29. Rudolf K, Cervinka M and Rudolf E: Dual inhibition of topoisomerases enhances apoptosis in melanoma cells. Neoplasma 57: 316-324, 2010.

30. Liu Y, Deng J, Wang L, et al: S1PR1 is an effective target to block STAT3 signaling in activated B cell-like diffuse large B-cell lymphoma. Blood 120: 1458-1465, 2012.

31. Dong YB, Yang HL, Elliott MJ and McMasters KM: Adenovirus-mediated E2F-1 gene transfer sensitizes melanoma cells to apoptosis induced by topoisomerase II inhibitors. Cancer Res 62: 1776-1783, 2002.

32. Kim D, Cheng GZ, Lindsley CW, Yang H and Cheng JQ: Targeting the phosphatidylinositol-3 kinase/Akt pathway for the treatment of cancer. Curr Opin Investig Drugs 6: 1250-1258, 2005.

33. Meier F, Schittek B, Busch S, et al: The RAS/RAF/MEK/ERK and PI3K/AKT signaling pathways present molecular targets for the effective treatment of advanced melanoma. Front Biosci 10: 2986-3001, 2005

34. Sinnberg T, Lasithiotakis K, Niessner H, et al: Inhibition of PI3K-AKT-mTOR signaling sensitizes melanoma cells to cisplatin and temozolomide. J Invest Dermatol 129: 1500-1515, 2009.

35. Liu X, Powlas J, Shi Y, et al: Rapamycin inhibits Akt-mediated oncogenic transformation and tumor growth. Anticancer Res 24: 2697-2704, 2004.

36. Sun SY, Rosenberg LM, Wang X, et al: Activation of Akt and eIF4E survival pathways by rapamycin-mediated mammalian target of rapamycin inhibition. Cancer Res 65: 7052-7208, 2005.

37. Francipane MG and Lagasse E: mTOR pathway in colorectal cancer: an update (Review). Oncotarget 15: 49-66, 2014

38. Rosa R, Marciano R, Malapelle U, et al: Sphingosine kinase 1 overexpression contributes to cetuximab resistance in human colorectal cancer models. Clin Cancer Res 19: 138-147, 2013. 